\title{
Blast overpressure induced axonal injury changes in rat brainstem and spinal cord
}

\author{
Srinivasu Kallakuri, Heena S. Purkait, Satya Dalavayi, Pamela VandeVord, John M. Cavanaugh
}

Department of Biomedical Engineering, Wayne State University, Detroit, MI 48201, USA

\begin{abstract}
Introduction: Blast induced neurotrauma has been the signature wound in returning soldiers from the ongoing wars in Iraq and Afghanistan. Of importance is understanding the pathomechansim(s) of blast overpressure (OP) induced axonal injury. Although several recent animal models of blast injury indicate the neuronal and axonal injury in various brain regions, animal studies related to axonal injury in the white matter (WM) tracts of cervical spinal cord are limited. Objective: The purpose of this study was to assess the extent of axonal injury in WM tracts of cervical spinal cord in male Sprague Dawley rats subjected to a single insult of blast OP. Materials and Methods: Sagittal brainstem sections and horizontal cervical spinal cord sections from blast and sham animals were stained by neurofilament light (NF-L) chain and beta amyloid precursor protein immunocytochemistry and observed for axonal injury changes. Results: Observations from this preliminary study demonstrate axonal injury changes in the form of prominent swellings, retraction bulbs, and putative signs of membrane disruptions in the brainstem and cervical spinal cord WM tracts of rats subjected to blast OP. Conclusions: Prominent axonal injury changes following the blast OP exposure in brainstem and cervical spinal WM tracts underscores the need for careful evaluation of blast induced injury changes and associated symptoms. NF-L immunocytochemistry can be considered as an additional tool to assess the blast OP induced axonal injury.
\end{abstract}

Key words: Axonal injury, blast overpressure, brainstem, neurofilament-light chain, spinal cord, traumatic brain injury

\section{Introduction}

An estimated 1.5 million US military personnel have been deployed to the conflicts in Iraq and Afghanistan since $2001^{[1]}$ and blast induced neurotrauma has been referred to as the signature wound of Operation Enduring Freedom $^{[2]}$ and Operation Iraqi Freedom (OIF) that accounts for over $60 \%$ of casualties. ${ }^{[3,4]}$ The continued stationing of service personnel in combat regions only adds to the risk of more people being exposed to blast and associated injuries. Improvements in preventative and management strategies have resulted in high survival rates while leaving many with invisible injuries affecting their quality of life. Although much of the ongoing

\section{Address for correspondence:}

Dr. Srinivasu Kallakuri, Department of Biomedical

Engineering, 818 W Hancock, Detroit, MI 48201, USA.

E-mail: skallakuri@wayne.edu

\begin{tabular}{|l|l|}
\hline \multicolumn{2}{|c|}{ Access this article online } \\
\hline Quick Response Code: & Website: \\
\hline & www.ruralneuropractice.com \\
\hline & \\
\hline
\end{tabular}

research interest has been directed at understanding injury changes in the brain, ${ }^{[5-21]}$ specific knowledge related to axonal injury changes in the brainstem and the spinal cord remain to be elucidated.

Blast injury can be categorized as, primary injury related to the shock wave overpressure (OP) and under pressure; secondary injury from blast-associated shrapnel; tertiary injury from being thrown out the by blast wind; and quaternary injury from a variety of physical processes associated with an explosive detonation. ${ }^{[22]}$ Of these, the mechanisms of primary injury following exposure to blast OP alone remains to be elucidated. The potential mechanisms by which blast OP can cause brain and other central nervous system injuries include: (a) direct interaction with the head by direct passage of the blast wave through the skull; (b) causing acceleration and/or rotation of the head; (c) transfer of energy from the blast

This is an open access article distributed under the terms of the Creative Commons Attribution-NonCommercial-ShareAlike 3.0 License, which allows others to remix, tweak, and build upon the work non-commercially, as long as the author is credited and the new creations are licensed under the identical terms.

For reprints contact: reprints@medknow.com

How to cite this article: Kallakuri S, Purkait HS, Dalavayi S, VandeVord P Cavanaugh JM. Blast overpressure induced axonal injury changes in rat brainstem and spinal cord. J Neurosci Rural Pract 2015;6:481-7. 
wave through large blood vessels in the abdomen and the chest to the central nervous system. ${ }^{[23]}$ These can cause both morphological and functional damage to distinct brain structures. ${ }^{[24]}$ Blast wave exposure was also shown to produce elevations of intracranial pressure in rats. ${ }^{[25]}$ It has been proposed that blast OP transmitted through the skull may also subject the brain to the same type of tensile and shear strains as seen in closed head injuries. ${ }^{[26]}$ Most recently, some experimental models have demonstrated that skull flexure and vibration occurring in response to a blast may contribute to injury. ${ }^{[25,27]}$ It was also suggested that blast induced axonal injury mechanisms were thought to be similar to mechanisms of diffuse axonal injury. ${ }^{[4]}$

The prevailing information related to blast induced axonal injuries comes from studies that demonstrated swollen neurons and myelin debris in the hippocampus after moderate blast injury in rats. ${ }^{[11]}$ Phosphorylated neurofilament-heavy chain ( $\mathrm{pNF}-\mathrm{H})$ accumulation in the neuronal perikarya of the cortex of rats following blast exposure (22.3 or 34.8 psi) was also reported. The pNF-H accumulation was attributed to a disturbed axonal transport machinery. ${ }^{[28]}$ On the other hand, beta amyloid precursor protein ( $\beta$-APP) immunostaining, a marker of impaired axoplasmic transport (IAT) ${ }^{[29]}$ revealed only mild axonal staining in the deep cerebellar white matter (WM) tracts. Recently, the utility of silver impregnation technique has gained prominence as shown by axonal pathology in the cortex and cerebellum of swine exposed to $55 \mathrm{psi}$ or $78 \mathrm{psi} \mathrm{OP},{ }^{[30]}$ and also in the deep cerebellar WM tracts and various brainstem regions of rats with body shielding exposed to single $\sim 35$ psi blast OP. ${ }^{[15]}$ Also reported was cytoskeletal damage in the cortex and hippocampus 7 days after the blast exposure using neurofilament immunocytochemistry. ${ }^{[31]}$ All these studies point to axonal injury changes in various regions of the brain at varying levels of blast OP and survival periods. Very limited evidence related to injury changes in the spinal cord comes from Czeiter et al. who demonstrated traumatic axonal injury in spinal WM tracts in an animal model of blunt traumatic brain injury (TBI) by $\beta$-APP and RMO14 immunohistochemistry. ${ }^{[32]}$ The exposure of blast OP (50.3 MPa), on the dorsal thoracic spinal cord (T9-T10) was also shown to induce necrosis and apoptosis in the lesion site in rats. ${ }^{[33]}$ Whether blast OP induced axonal changes observed in brain would also be observed in cervical spinal cord WM tracts remains to be investigated.

Thus, the purpose of this study was to assess the extent of axonal injury in WM tracts of the cervical spinal cord in rats exposed to blast OP in a cephalic-to-caudal orientation. Results from this preliminary study for the first time demonstrate axonal injury changes in the form of swellings, retraction balls, vacuolated axons, and more importantly as putative membrane disruptions in cervical spinal cord WM tracts. Our results also support the presence of such axonal injury changes in various WM tracts of the brainstem.

\section{Materials and Methods}

Blast overpressure induction: All procedures were approved by the Institutional Animal Care and Use Committee. Male Sprague Dawley rats (250-275 g, Harlan) were exposed to a single insult of blast OP. Briefly, animals without any chest protection were anesthetized (Isoflurane $3 \%$ and $0.6 \mathrm{~L} /$ min oxygen). They were harnessed to a sled to reduce the dynamic pressure load and prevent the translation of the entire body. Rats were positioned inside the lexan section $45^{\prime \prime}$ from the open end of the shock tube with a cephalic-to-rostral orientation toward the shock wave.

The shock front and the dynamic OP were generated by a custom-built shock tube (Figure 1; 12" diameter, 193" long shock-producing tube, ORA Inc., Fredericksburg, VA). The shock tube pressure was determined by three
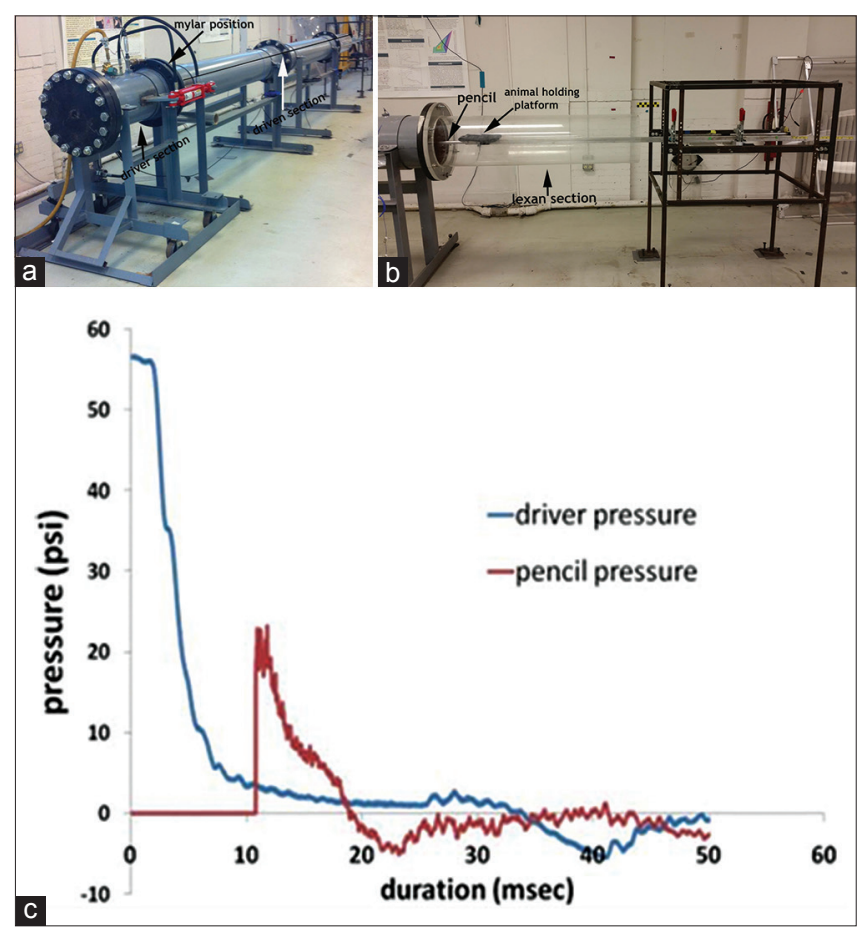

Figure 1: (a) The shock tube used to simulate a free-field shock wave. Also shown are the driver and driven section, with the position for placing calibrated Mylar sheets being indicated (b) the lexan section for visualization of blast pressure pencil probe and the animal holding platform. The aluminum rod was secured to the dynamic sled supported by the metal frame (c) graph shows a typical driver pressure profile (blue) and static OP at the rat head as detected by the pencil probe (red) indicating the generation of the pressure wave 
sensors placed within the tube (2.4 m apart) and the static $\mathrm{OP}$ at the rat was measured with a pencil probe (PCB Piezotronics) housed in an aluminium rod that acts as a support for the rat holding platform. Desired peak static OPs were produced with compressed helium and calibrated Mylar sheets (GE Richards Graphics Supplies Inc., Landsville, PA) to simulate a free field blast wave. ${ }^{[25]}$ Pressure measurements were collected at $250 \mathrm{kHz}$ using a Dash 8HF data acquisition system (Astro-Med, Inc., West Warwick, RI).

A total of 32 male Sprague Dawley rats were used in this investigation. Before determining cervical spinal cord axonal injury changes, a preliminary investigation of axonal injury changes in brainstem WM tracts was performed. For this purpose, 14 rats were exposed to blast OP in the range of 12 psi $(n=5), 16$ psi $(n=4)$, or 22 psi $(n=5)$ and were allowed to survive for either 24 or $48 \mathrm{~h}$. It was reasoned that axonal injury changes observed in the brainstem WM tracts would extend to cervical spinal cord WM tracts also. For studying axonal injury changes in the cervical spinal cord WM tracts, 12 rats were subjected to a single insult of blast OP in the range of 22 psi. The pressure of 22 psi was used based on the apparent axonal injury changes observed in the brainstems of rats subjected to this pressure. These rats were separated into groups that survived for $6 \mathrm{~h}(n=6)$ or $24 \mathrm{~h}(n=6)$. These rats were also monitored for the duration of surface righting, an indirect indicator of loss of consciousness. Sham $(n=6)$ rats were subjected to identical experimental procedures but were not exposed to blast OP.

\section{Termination and fixation}

After their respective survival period, each rat was euthanized (sodium pentobarbital $120 \mathrm{mg} / \mathrm{kg}$, intra peritoneal) and then transcardially perfused with cold $4 \%$ paraformaldehyde in phosphate buffered saline $(0.2$ M PBS, pH 7.4) after which the brain and C2-C7 cervical spinal cord segment were removed and post fixed (4\% paraformaldehyde in 30\% sucrose). For brainstem sectioning, a midline longitudinal incision was made on the ventral aspect of the brainstem separating it into two sagittal halves. One half of the brainstem region was sagitally cut into $40 \mu \mathrm{m}$ thick frozen sections that were serially collected in $1 \times$ PBS filled multi-well plates. The entire segment of the $\mathrm{C} 2-\mathrm{C} 7$ cervical spinal cord was sectioned horizontally into $40 \mu \mathrm{m}$ thick frozen sections and collected separately in $1 \times$ PBS filled multi-well plates.

\section{Neurofilament light-chain and beta amyloid precursor protein immunohistochemistry}

Five serial sagittal sections of brainstem from the midline to $1000 \mu \mathrm{m}$ lateral and four horizontal cervical spinal cord sections from all the blast and sham specimens were subjected to neurofilament light chain (NF-L) and $\beta$-APP immunohistochemistry. Briefly, the sections were subjected to antigen retrieval by incubation in a citrate buffer ( $\mathrm{pH}$ 6.0) at $90^{\circ} \mathrm{C}$ for $1 \mathrm{~h}$. Then they were immersed for $1 \mathrm{~h}$ in $0.3 \%$ hydrogen peroxide to quench endogenous peroxidase activity. This was followed by overnight incubation either in a polyclonal rabbit anti-NF-L antibody (1:500, Cat\#AB9568; Millipore, Temecula CA) or anti $\beta$-APP antibody (1:250, Cat \# 51-2700, Life Technologies, Grand Island, NY) diluted in $2 \%$ normal goat serum (Vector Laboratories, Burlingame, CA) in $1 \%$ bovine serum albumin. The following day, sections were re-incubated in a 1:250 dilution of goat biotinylated antirabbit IgG (Vector Laboratories, Burlingame, CA) followed by exposure to Vectastain Elite $A B C$ reagent. Finally, the peroxidase activity was developed by brief incubation in 3, 3'-diaminobenzidine and hydrogen peroxide. The sections were washed, dehydrated, and cover slipped using permount. In the control incubations, normal goat serum was substituted for primary antibody. Brainstem sections harvested from rats subjected to severe TBI by an impact acceleration device ${ }^{[34]}$ were used as positive control.

\section{Light microscopy and qualitative analysis}

All the stained sections were observed under a light microscope (Leica DMLB, Leica Microsystems Ltd., Heerburg, Switzerland) to visualize NF-L and $\beta$-APP reactive axonal injury changes. Digital images of NF-L and $\beta$-APP reactive axons in various brainstem and cervical spinal cord regions were obtained by a digital camera system (ProgRes C7, JENOPTIK Laser Optik Systeme, $\mathrm{GmbH}$ ) and taken at a single focal plane.

\section{Results}

\section{Axonal injury changes in the brainstem}

Brainstem and cervical spinal cord sections from sham rats revealed NF-L reactive axonal profiles in various WM tracts. The axons could be seen coursing through the WM tracts of the brainstem and cervical spinal cord uninterrupted for extended lengths. In the brainstem sections of sham animals, the NF-L reactivity was observed to be distributed throughout the core of the axon cylinder [Figure 2a]. Axons appeared to be with uniform caliber with no apparent lobulations or retraction balls. On the other hand, sections from the positive control (brainstem sections from rats subjected to severe TBI) showed prominent axonal swellings with vacuolations and retraction balls [Figure 2b] supporting the capability of NF-L immunohistochemistry to reveal the injured axons. 


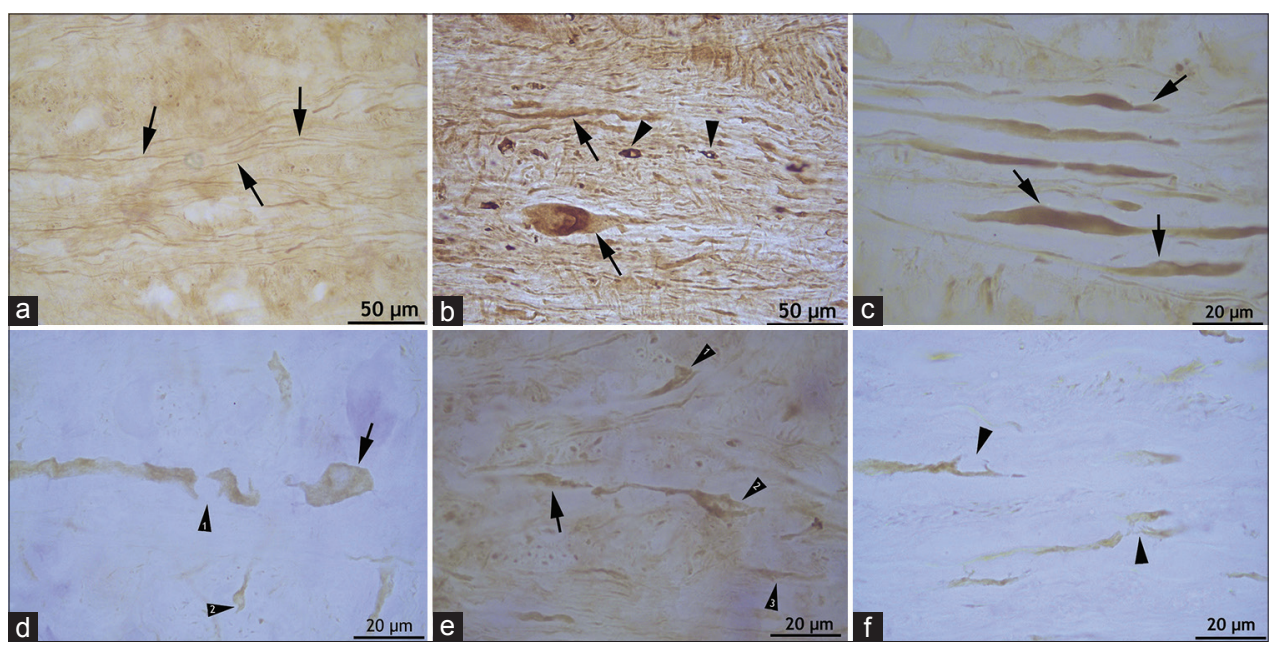

Figure 2: (a) Normal looking axons with uniform caliber in brainstem sections from a sham $48 \mathrm{~h}$ survival animal (b) positive control section from brainstem of a rat subjected to closed head traumatic brain injury by an impact acceleration device showing prominent swellings and retraction balls (arrow), and retraction balls with vacuolations (arrow heads) by neurofilament light chain immunohistochemistry (c) abnormal swollen axons (arrows) in the brainstem white matter tracts following exposure to a single insult of 16 psi blast overpressure. Such swollen axons were also observed in the brainstems of animals exposed to 22 psi blast overpressure (d) a representative axon showing axonal enlargements with vacuolations (arrowhead) and a terminal retraction bulb (arrow) in the pyramidal tract in the brainstem of a rat exposed to a single insult of 22 psi blast overpressure (e and f) putative membrane disruptions (arrowheads) in the brainstem sections of a rat subjected to 16 psi and 22 psi blast overpressure respectively

Brainstem sections from rats exposed to blast OP revealed very prominent NF-L reactive axon profiles. Qualitatively, these changes were prominently revealed by NF-L reactive focal accumulations that correlated with changes in the axonal diameter. Such focally swollen and enlarged axons could be found in all brainstem sections from all the blast animals. Brainstem sections from the 12 psi OP group showed axonal morphology changes in the fibers of pyramidal decussation, transverse fibers in the region of pons, medial lemniscus and other areas of reticular formation in the form of swollen axonal profiles, retraction balls, and axons with vacuolations from among a population of axons with uniform caliber.

Although this was a qualitative study, the incidence of swollen axons, retraction balls, and vacuolated axons appeared to be more prominent in the brainstem sections from the $16 \mathrm{psi}$ [Figure 2c] and 22 psi OP group [Figure 2d] than in sections from the 12 psi OP group. These NF-L reactive injury profiles were particularly found in the large diameter fibers of the medial lemniscus and the medial longitudinal fasciculus and were seen occasionally in the thin fibers of the pyramidal tracts. NF-L reactive injured axons in the 16 psi and 22 psi group also appeared to be more convoluted. Vacuolated areas in sections from 16 psi and 22 psi groups were indicated by areas free of NF-L staining in an otherwise labeled axoplasmic core [Figure 2d]. Also observed were axons with multiple bulbous dilations particularly in brainstem sections from 16 psi [Figure 2c] and 22 psi more so than in sections from 12 psi. One interesting observation from this study was the presence of interruptions in the axonal membrane [Figure 2e and $\mathrm{f}$ ]. Interruptions in the axonal membrane indicated by discontinuity or spacing in the axonal membrane occasionally accompanied by extended filamentous profiles [Figure $2 \mathrm{f}$ ] was observed. Such membrane disruptions were found more prominently in brainstem sections from both 16 psi and 22 psi blast exposure compared to those from the $12 \mathrm{psi}$ exposure group. No prominent $\beta$-APP reactive axons were found in the brainstem WM tracts.

\section{Axonal injury changes in cervical spinal cord}

In the cervical spinal cord of sham animals, normal looking axons with uniform caliber were observed in the WM tracts [Figure 3a]. On the other hand, in the cervical spinal cord of blast exposed rats, axonal injury changes similar to those seen in the brainstem were found. NF-L reactive swollen axons with altered caliber, vacuolations, and retraction balls could be observed in various $\mathrm{WM}$ tracts at both $6 \mathrm{~h}$ [Figure $3 \mathrm{~b}$ and c] and $24 \mathrm{~h}$ [Figure 3d and e] survival periods. Vacuolations in the axons appeared to be located in the core of the axon [Figure 3b] with some appearing as semilunar spaces [Figure 3e]. Similar to brainstem injury changes, these injured axons could be observed predominantly in the large caliber axons of the cervical spinal cord WM tracts. These injury changes were observed in sections extending from the dorsal to ventral aspects of the spinal cord. One striking observation from studying the cervical spinal cord horizontal sections is the prevalence and the prominence of axonal injury in the form of putative membrane disruptions [Figure $3 \mathrm{c}$ and $\mathrm{d}$ ] in large caliber axons similar to those seen in the brainstem. Disrupted 


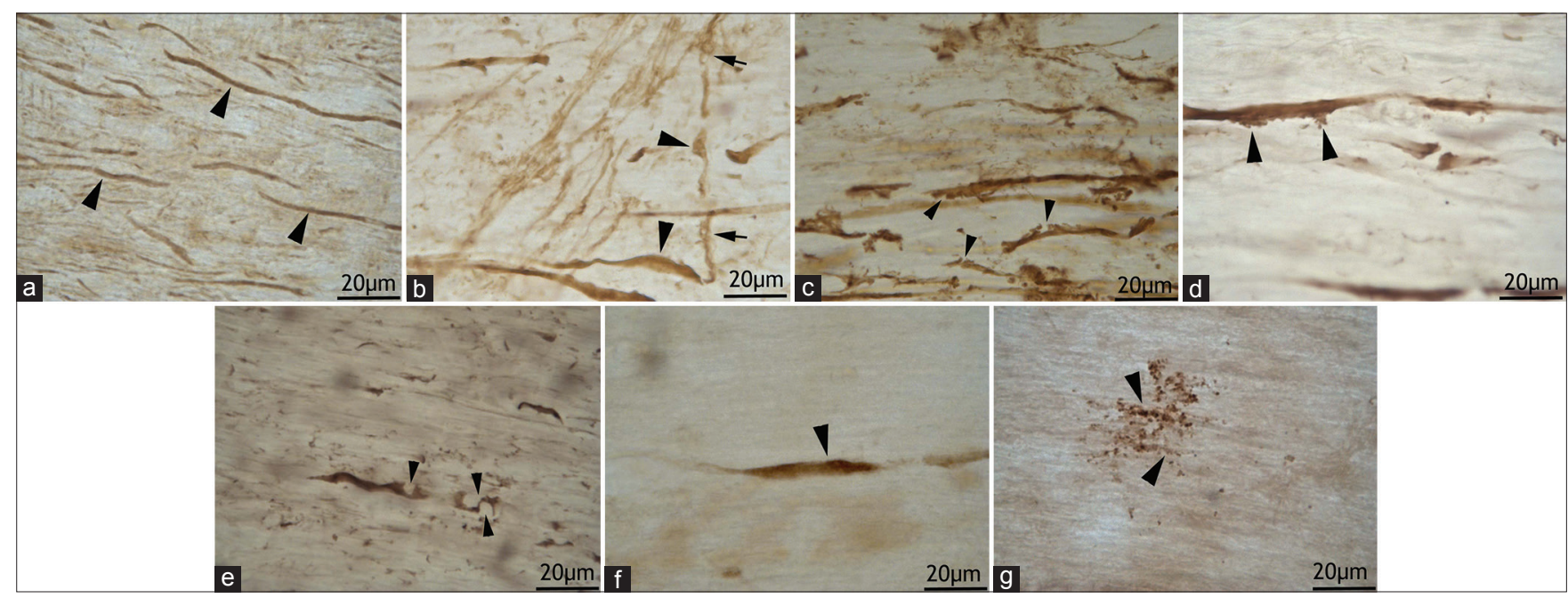

Figure 3: (a) Normal looking axons in the white matter tracts of cervical spinal cord of a $24 \mathrm{~h}$ survival sham animal (b) axons with prominent swellings (arrowheads) and swollen axons with vacuolations (arrows) in the white matter tracts of cervical spinal cord at $6 \mathrm{~h}$ (c and d) prominent disruptions of axonal membranes (arrowheads) at $6 \mathrm{~h}$ and $24 \mathrm{~h}$ respectively. These disruptions in the form of wide spaces on the margins or projections with ragged edges could be seen in various large caliber axons (e) axonal injury in the form of vacuolations in the axonal core at $24 \mathrm{~h}(\mathrm{f})$ a beta amyloid precursor protein reactive swollen axon in the cervical spinal cord white matter tract at $6 \mathrm{~h}$ post blast period $(\mathrm{g})$ beta amyloid precursor protein reactive punctate axons $24 \mathrm{~h}$ post blast

axons showing membranes with extended filamentous profiles and ragged edges in otherwise normal looking axons could be seen in spinal cord sections at both 6 $\mathrm{h}$ and $24 \mathrm{~h}$ survival periods. $\beta$-APP immunoreactive axon profiles in the form of axonal swellings and axonal retraction balls could be found as early as $6 \mathrm{~h}$ as well as $24 \mathrm{~h}$ post blast in the cervical spinal cord [Figure $3 \mathrm{f}$ ]. $\beta$-APP reactive large caliber axons with occasional vacuoles and putative membrane disruptions were also observed. One additional observation is the presence of $\beta$-APP reactive micro-beaded profiles in the cervical spinal cord WM tracts [Figure $3 g$ ]. These $\beta$-APP reactive micro beaded profiles were observed as parallel lines or as small punctate clusters of reactive products.

\section{Discussion}

This study is the first to provide evidence of axonal injury changes in the brainstem as well as in the cervical spinal cord of rats subjected to a single insult of blast OP in a cephalic-to-caudal orientation. In this study, we observed primarily in the large caliber axons, prominent NF-L reactive axonal injury profiles in the form of swollen axons, retraction balls, axons with vacuolations and most importantly axons with signs of membrane disruptions in the WM tracts of both the brain stem and cervical spinal cord. Occasionally, the vacuolated appearance of the axons [Figures $2 \mathrm{~d}$ and $3 \mathrm{e}$ ] was reminiscent of axons undergoing neurofilament compaction, one of the components in the pathology of traumatic axonal injury as revealed previously from studies by Stone et al. ${ }^{[35]}$ Also observed were $\beta$-APP reactive swollen axons in the cervical spine $\mathrm{WM}$ tracts at both $6 \mathrm{~h}$ and $24 \mathrm{~h}$ post blast, especially in the large caliber axons. The observation of $\beta$-APP reactive punctate profiles with some of them as parallel lines or as small clusters in the cervical spinal cord WM tracts may be indicative of disruption of small caliber axons as well. Taken together, these findings may suggest that large and small caliber axons may be undergoing diverse injury changes. The presence of axonal injury changes at $6 \mathrm{~h}$ post blast indicates the initiation of these injury changes soon after the blast. Furthermore, the prominent observation of membrane disruption changes in large caliber axons may also suggest that blast OP induces axonal injury changes different from a blunt TBI. The extent of these putative membrane disruptions may need further characterization. Also noticed was a prolonged surface righting duration in animals exposed to blast OP $(203.1 \pm 78.1 \mathrm{~s})$ compared to sham animals $(90.6 \pm 29.9 \mathrm{~s})$.

Previous studies demonstrating apparent blast OP induced axonal injury changes especially in the cervical spinal cord WM tracts are nonexistent. However, various prior studies showed axonal injury in diverse brain regions with a very few reports on changes in the brainstem. Using a shock tube system, Koliatsos et al. have shown the axonal injury in the form of $\beta$-APP swellings and retraction balls in mice corpus callosum 7 days after the blast exposure with no apparent reactivity at earlier time points. ${ }^{[14]}$ This is similar to our observation of no $\beta$-APP reactive axons in the brainstem sections. However, we show the utility of NF-L immunohistochemistry which was previously 
used to investigate the axonal injury following TBI. ${ }^{[36]}$ So far, the most widely used histochemical procedure to assess the blast induced axonal injury has been the silver impregnation technique that revealed axonal injury changes in deep cerebellar WM, peduncles, corticospinal tract, and visual pathway in animals subjected to blast OP of 26.5 psi described as mild intensity. ${ }^{[14]}$ In the current study, we show brainstem injury changes after expsoure to a single blast OP exposure $(12,16$ or 22 psi) with changes in spinal cord also being shown after exposure to single insult of 22 psi blast OP.

In another investigation by Garman et al., rats subjected to blast OP (35 psi) resulted in a $25 \%$ mortality and axonal degeneration was evidenced by amino cupric silver staining in the deep cerebellar WM regions prominently at $24 \mathrm{~h}$ and $72 \mathrm{~h}$ with $\beta$-APP stained sections showing only minimal axonal staining at $24 \mathrm{~h}$ in the deep cerebellar WM and suggetsed that amino cupric staining as more sensitive than $\beta$-APP immunohistochemistry. ${ }^{[15]}$ In a recent investigation, Risling et al. 2011 suggested that the blast model employed has similarities to mild TBI but showed no apparent diffuse axonal injury and cell death after blast (19-38 psi) but stated that others using the same device demonstrated degenerative changes in rat brains exposed to the same tube. ${ }^{[21]}$ Svetlov et al. 2011 also showed axonal injury by prominent silver staining in deep brain areas such as the caudal diencephalon at $48 \mathrm{~h}$ with prominent silver accumulation in the perivascular spaces and sub-ventricular zones at $48 \mathrm{~h}$ and predominant tissue localization at 5 days after blast. ${ }^{[9]}$ Such nerve injury by silver staining was also shown by Bauman et al. in the WM tracts of superior corona radiata and the ipsilateral cerebellum. ${ }^{[30]}$ Other injury forms such as expanded perineural spaces, increased number of cytoplasmic vacuoles and formation of laminal bodies were observed at $24 \mathrm{~h}$ and 5 days after a whole body blast injury. ${ }^{[11]}$

On the other hand, neurofilament immunohistochemistry was also used by others to study blast induced axonal injury. In rats exposed to 22 or 34 psi OP, pNF-H immunoreactivity was observed in neuronal perikarya of layers II-IV (temporal cortex) at $18 \mathrm{~h}$ after 34 psi blast exposure. The frequency of pNF-H positive perikarya increased from $18 \mathrm{~h}$ to $48 \mathrm{~h}$ to a level that remained high at 7 days and subsided by 21 days. Exposure to 22 psi blast OP was reported to induce a similar redistribution of p-NFH immunoreactivity in the cerebral cortex and hippocampus. The authors suggested dephosphorylation of NF-H in the axons and dendrites and a piling of pNF-H in the perikarya due to a disturbed axonal transport. ${ }^{[28,37]}$ Such a disturbed axonal transport is shown by the current investigation in the form of NF-L reactive swellings and RB with little reactivity from $\beta$-APP. Furthermore, it was previously suggested that proteolysis may contribute to reduced $\mathrm{pNF}-\mathrm{H}^{[36]}$ or activation of calpains. ${ }^{[38]}$ Spot like accumulations of the light subunit of the NF which also suggests impaired axonal transport leading to axonal swellings which has also been shown in various brain trauma models. ${ }^{[36,39,40]}$ Other investigators have shown a decrease in protein levels of both NF-L and NF-H after TBI suggesting specific biochemical alterations of the neuronal cytoskeleton after brain injury and such alterations are also possible following blast OP but were not investigated. ${ }^{[41]}$

What are the potential clinical implications of these observed changes? An assessment of OIF and Operation Enduring Freedom soldiers revealed that $70 \%$ of soldiers experience pain in more than one site including the head, lower back (46.4\%), lower limbs (31.3\%), upper limbs $(7.5 \%)$ neck $(6 \%)$, shoulders, face, and the abdomen. ${ }^{[42]}$ Their treatment challenge requires a detailed understanding of the cellular processes that may underlie various conditions especially after blast injury, which may also be associated with TBI. It has been estimated that more than $30 \%$ of service members with severe TBI and more than $40 \%$ with moderate TBI, also sustained concomitant injuries to both the upper and lower extremities and a high proportion of spine and back injuries. ${ }^{[31]}$ Furthermore, patients referred for treatment of chronic pain were found to have a history and symptoms indicative of an untreated $\mathrm{TBI}^{[43]}$ and also reported to have neck/shoulder, back, upper limb, and lower limb pain. ${ }^{[4]}$ While the current study highlights the presence of axonal injury changes following blast exposure, other changes such as glial proliferation and inflammation also occur (unpublished data). Therefore, it is important to characterize some of the cellular injury changes following blast exposure so that neurodegenerative changes are better understood.

\section{Financial support and sponsorship}

North American Spine Society, United States Office of Naval Research Award N000140810585.

\section{Conflicts of interest}

There are no conflicts of interest.

\section{References}

1. Hoge CW, McGurk D, Thomas JL, Cox AL, Engel CC, Castro CA. Mild traumatic brain injury in U.S. Soldiers returning from Iraq. N Engl J Med 2008;358:453-63.

2. Ihling C, Szombathy T, Bohrmann B, Brockhaus M, Schaefer HE, 
Loeffler BM. Coexpression of endothelin-converting enzyme-1 and endothelin-1 in different stages of human atherosclerosis. Circulation 2001;104:864-9.

3. Ling G, Bandak F, Armonda R, Grant G, Ecklund J. Explosive blast neurotrauma. J Neurotrauma 2009;26:815-25.

4. Elder GA, Cristian A. Blast-related mild traumatic brain injury: Mechanisms of injury and impact on clinical care. Mt Sinai J Med 2009;76:111-8.

5. Cernak I, Merkle AC, Koliatsos VE, Bilik JM, Luong QT, Mahota TM, et al. The pathobiology of blast injuries and blast-induced neurotrauma as identified using a new experimental model of injury in mice. Neurobiol Dis 2011;41:538-51.

6. Long JB, Bentley TL, Wessner KA, Cerone C, Sweeney S, Bauman RA. Blast overpressure in rats: Recreating a battlefield injury in the laboratory. J Neurotrauma 2009;26:827-40.

7. Svetlov SI, Larner SF, Kirk DR, Atkinson J, Hayes RL, Wang KK. Biomarkers of blast-induced neurotrauma: Profiling molecular and cellular mechanisms of blast brain injury. J Neurotrauma 2009;26:913-21.

8. Svetlov SI, Prima V, Glushakova O, Svetlov A, Kirk DR, Gutierrez H, et al. Neuro-glial and systemic mechanisms of pathological responses in rat models of primary blast overpressure compared to "composite" blast. Front Neurol 2012;3:15.

9. Svetlov SI, Prima V, Kirk DR, Gutierrez H, Curley KC, Hayes RL, et al. Morphologic and biochemical characterization of brain injury in a model of controlled blast overpressure exposure. J Trauma 2010;69:795-804.

10. Cernak I, Wang Z, Jiang J, Bian X, Savic J. Cognitive deficits following blast injury-induced neurotrauma: Possible involvement of nitric oxide. Brain Inj 2001;15:593-612.

11. Cernak I, Wang Z, Jiang J, Bian X, Savic J. Ultrastructural and functional characteristics of blast injury-induced neurotrauma. J Trauma 2001;50:695-706.

12. Park E, Gottlieb JJ, Cheung B, Shek PN, Baker AJ. A model of low-level primary blast brain trauma results in cytoskeletal proteolysis and chronic functional impairment in the absence of lung barotrauma. J Neurotrauma 2011;28:343-57.

13. Pun PB, Kan EM, Salim A, Li Z, Ng KC, Moochhala SM, et al. Low level primary blast injury in rodent brain. Front Neurol 2011;2:19.

14. Koliatsos VE, Cernak I, Xu L, Song Y, Savonenko A, Crain BJ, et al. A mouse model of blast injury to brain: Initial pathological, neuropathological, and behavioral characterization. J Neuropathol Exp Neurol 2011;70:399-416.

15. Garman RH, Jenkins LW, Switzer RC $3^{\text {rd }}$, Bauman RA, Tong LC, Swauger PV, et al. Blast exposure in rats with body shielding is characterized primarily by diffuse axonal injury. J Neurotrauma 2011;28:947-59.

16. Wang Y, Wei Y, Oguntayo S, Wilkins W, Arun P, Valiyaveettil M, et al. Tightly coupled repetitive blast-induced traumatic brain injury: Development and characterization in mice. J Neurotrauma 2011;28:2171-83.

17. Säljö A, Mayorga M, Bolouri H, Svensson B, Hamberger A. Mechanisms and pathophysiology of the low-level blast brain injury in animal models. Neuroimage 2011;54 Suppl 1:S83-8.

18. Säljö A, Bolouri H, Mayorga M, Svensson B, Hamberger A. Low-level blast raises intracranial pressure and impairs cognitive function in rats: Prophylaxis with processed cereal feed. J Neurotrauma 2010;27:383-9.

19. Sajja VS, Galloway MP, Ghoddoussi F, Thiruthalinathan D, Kepsel A, Hay K, et al. Blast-induced neurotrauma leads to neurochemical changes and neuronal degeneration in the rat hippocampus. NMR Biomed 2012;25:1331-9.

20. Vandevord PJ, Bolander R, Sajja VS, Hay K, Bir CA. Mild neurotrauma indicates a range-specific pressure response to low level shock wave exposure. Ann Biomed Eng 2012;40:227-36.

21. Risling M, Plantman S, Angeria M, Rostami E, Bellander BM, Kirkegaard M, et al. Mechanisms of blast induced brain injuries, experimental studies in rats. Neuroimage 2011;54 Suppl 1:S89-97.

22. Moore DF, Jaffee MS. Military traumatic brain injury and blast. NeuroRehabilitation 2010;26:179-81.
23. Courtney AC, Courtney MW. A thoracic mechanism of mild traumatic brain injury due to blast pressure waves. Med Hypotheses 2009;72:76-83.

24. Cernak I, Savic J, Ignjatovic D, Jevtic M. Blast injury from explosive munitions. J Trauma 1999;47:96-103.

25. Leonardi AD, Bir CA, Ritzel DV, VandeVord PJ. Intracranial pressure increases during exposure to a shock wave. J Neurotrauma 2011;28:85-94.

26. Kocsis JD, Tessler A. Pathology of blast-related brain injury. J Rehabil Res Dev 2009;46:667-72.

27. Bolander R, Mathie B, Bir C, Ritzel D, VandeVord P. Skull flexure as a contributing factor in the mechanism of injury in the rat when exposed to a shock wave. Ann Biomed Eng 2011;39:2550-9.

28. Säljö A, Bao F, Haglid KG, Hansson HA. Blast exposure causes redistribution of phosphorylated neurofilament subunits in neurons of the adult rat brain. J Neurotrauma 2000;17:719-26.

29. Stone JR, Okonkwo DO, Dialo AO, Rubin DG, Mutlu LK, Povlishock JT, et al. Impaired axonal transport and altered axolemmal permeability occur in distinct populations of damaged axons following traumatic brain injury. Exp Neurol 2004;190:59-69.

30. Bauman RA, Ling G, Tong L, Januszkiewicz A, Agoston D, Delanerolle N, et al. An introductory characterization of a combat-casualty-care relevant swine model of closed head injury resulting from exposure to explosive blast. J Neurotrauma 2009;26:841-60.

31. MacGregor AJ, Dougherty AL, Galarneau MR. Injury-specific correlates of combat-related traumatic brain injury in Operation Iraqi Freedom. J Head Trauma Rehabil 2011;26:312-8.

32. Czeiter E, Pal J, Kovesdi E, Bukovics P, Luckl J, Doczi T, et al. Traumatic axonal injury in the spinal cord evoked by traumatic brain injury. J Neurotrauma 2008;25:205-13.

33. Wang Y, Ye Z, Hu X, Huang J, Luo Z. Morphological changes of the neural cells after blast injury of spinal cord and neuroprotective effects of sodium beta-aescinate in rabbits. Injury 2010;41:707-16.

34. Kallakuri S, Li Y, Zhou R, Bandaru S, Zakaria N, Zhang L, et al. Impaired axoplasmic transport is the dominant injury induced by an impact acceleration injury device: An analysis of traumatic axonal injury in pyramidal tract and corpus callosum of rats. Brain Res 2012;1452:29-38.

35. Stone JR, Singleton RH, Povlishock JT. Intra-axonal neurofilament compaction does not evoke local axonal swelling in all traumatically injured axons. Exp Neurol 2001;172:320-31.

36. Povlishock JT, Marmarou A, McIntosh T, Trojanowski JQ, Moroi J. Impact acceleration injury in the rat: Evidence for focal axolemmal change and related neurofilament sidearm alteration. J Neuropathol Exp Neurol 1997;56:347-59.

37. Povlishock JT, Pettus EH. Traumatically induced axonal damage: Evidence for enduring changes in axolemmal permeability with associated cytoskeletal change. Acta Neurochir Suppl 1996;66:81-6.

38. Saatman KE, Graham DI, McIntosh TK. The neuronal cytoskeleton is at risk after mild and moderate brain injury. J Neurotrauma 1998;15:1047-58.

39. Maxwell WL, Graham DI. Loss of axonal microtubules and neurofilaments after stretch-injury to Guinea pig optic nerve fibers. J Neurotrauma 1997;14:603-14.

40. Yaghmai A, Povlishock J. Traumatically induced reactive change as visualized through the use of monoclonal antibodies targeted to neurofilament subunits. J Neuropathol Exp Neurol 1992;51:158-76.

41. Posmantur R, Hayes RL, Dixon CE, Taft WC. Neurofilament 68 and neurofilament 200 protein levels decrease after traumatic brain injury. J Neurotrauma 1994;11:533-45.

42. Gironda RJ, Clark ME, Massengale JP, Walker RL. Pain among veterans of Operations Enduring Freedom and Iraqi Freedom. Pain Med 2006; 7:339-43.

43. Andary MT, Crewe N, Ganzel SK, Haines-Pepi C, Kulkarni MR, Stanton DF, et al. Traumatic brain injury/chronic pain syndrome: A case comparison study. Clin J Pain 1997;13:244-50.

44. Lahz S, Bryant RA. Incidence of chronic pain following traumatic brain injury. Arch Phys Med Rehabil 1996;77:889-91. 